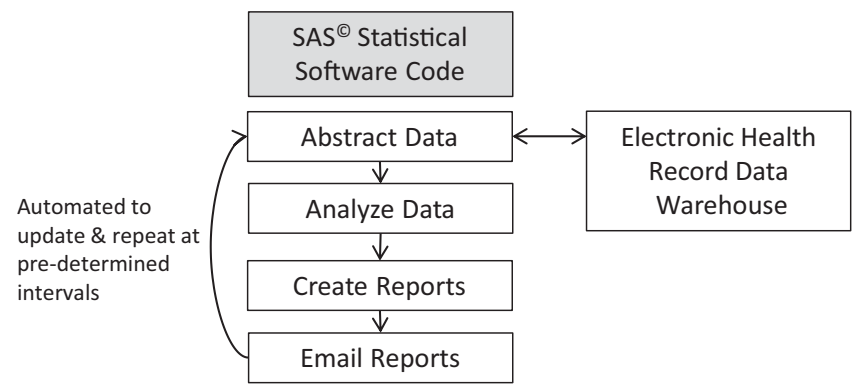

Fig. 1. The $\mathrm{OASIS}^{\odot}$ method for automating the creation of individualized provider antibiotic prescribing reports with peer comparison.

which OASIS $^{\odot}$-generated reports were a major component, reduced unnecessarily long durations of antibiotics for uncomplicated AOM by $76 \%$ in 6 months. We expect that minimal data analyst time would be needed to modify the code to mimic the project at another organization.

Given the simplicity and flexibility of this approach, we encourage other organizations to utilize this method. The small amount of time and small financial investment at the beginning of the intervention was overwhelmingly justified by the reduced time and cost over the life of the project. We plan to collaborate with the University of Rochester to make minor modifications to the EHR to link antibiotic orders to a diagnosis and to set up OASIS $^{\odot}$ for their antibiotic stewardship needs. In conclusion, OASIS $^{\odot}$ is a potentially important tool for streamlining tracking and reporting. Organizations should consider adopting OASIS ${ }^{\odot}$ as a means to improve efficiency of outpatient antibiotic stewardship.

Supplementary material. To view supplementary material for this article, please visit https://doi.org/10.1017/ice.2020.1409

Financial support. Support for OASIS ${ }^{\odot}$ was provided by the Denver Health Office of Research Pilot Grant Program. H.F. received salary support from the Eunice Kennedy Shriver National Institute of Child Health \& Human Development of the National Institutes of Health (grant no. K23HD099925).
The content is solely the responsibility of the authors and does not necessarily represent the official views of the National Institutes of Health.

Conflicts of interest. The authors have no conflicts of interest to disclose.

Note. OASIS ${ }^{\oplus}$ may be licensed at no cost by completing a web-based license or by contacting the lead author (Holly Frost) or coauthors.

\section{References}

1. Centers for Disease Control and Prevention. Antibiotic Resistance Threats in the United States, 2019. Atlanta, GA: US Department of Health and Human Services, CDC; 2019.

2. Bates DW, Cullen DJ, Laird N, et al. Incidence of adverse drug events and potential adverse drug events: implications for prevention. ADE Prevention Study Group. JAMA 1995;274:29-34.

3. Mohsen S, Dickinson JA, Somayaji R. Update on the adverse effects of antimicrobial therapies in community practice. Medecin de Famille Canadien 2020;66:651-659.

4. Brown KA, Khanafer N, Daneman N, Fisman DN. Meta-analysis of antibiotics and the risk of community-associated Clostridium difficile infection. Antimicrob Agents Chemother 2013;57:2326-2332.

5. Ahmad OF, Akbar A. Microbiome, antibiotics and irritable bowel syndrome. Br Med Bull 2016;120:91-99.

6. Horton DB, Scott FI, Haynes K, et al. Antibiotic exposure and juvenile idiopathic arthritis: a case-control study. Pediatrics 2015;136:e333-e343.

7. Fleming-Dutra KE, Hersh AL, Shapiro DJ, et al. Prevalence of inappropriate antibiotic prescriptions among US ambulatory care visits, 2010-2011. JAMA 2016;315:1864-1873.

8. Sanchez GV, Fleming-Dutra KE, Roberts RM, Hicks LA. Core elements of outpatient antibiotic stewardship. Morbid Mortal Wkly Rep 2016; 65:1-12.

9. Frost HM, Andersen LM, Fleming-Dutra KE, Norlin C, Czaja CA. Sustaining outpatient antimicrobial stewardship: do we need to think further outside the box? Infect Control Hosp Epidemiol 2020;41:382-384.

10. Frost HM, Lou Y, Munsiff S. OASIS (Outpatient Automated Stewardship Information System). IDWeek IDEA Incubator conference held virtually; October 2020. OASIS Wins Grand Prize at IDea Incubator Competition Held in Collaboration with Johnson \& Johnson Innovation - JLABS. https://www.idsociety.org/news-publications-new/articles/2020/oasiswins-grand-prize-at-idea-incubator-competition-held-in-collaborationwith-johnson-johnson-innovation-jlabs/

\title{
Association between extended-spectrum $\beta$-lactamase-producing Escherichia coli and oral third-generation cephalosporins
}

\author{
Hiroki Hosokawa $\mathrm{BS}^{1}$ (1), Satoru Mitsuboshi $\mathrm{PhD}^{2}$ (1), Hirokazu Isobe $\mathrm{PhD}^{3}$, Kenichi Kobayashi ${ }^{4}$, Hiroshi Moro MD, $\mathrm{PhD}^{5}$ \\ and Toshiaki Kikuchi MD, $\mathrm{PhD}^{5}$ \\ ${ }^{1}$ Department of Pharmacy, Agano City Hospital, Niigata, Japan, ${ }^{2}$ Department of Pharmacy, Kaetsu Hospital, Niigata, Japan, ${ }^{3}$ Department of Pharmacy, Niigata \\ University Medical and Dental Hospital, Niigata, Japan, ${ }^{4}$ Department of Pharmacy, Nagaoka Red Cross Hospital, Niigata, Japan and ${ }^{5}$ Department of Respiratory \\ Medicine and Infectious Disease, Niigata University Graduate School of Medical and Dental Sciences, Niigata, Japan
}

\footnotetext{
Author for correspondence: Satoru Mitsuboshi, E-mail: mitsuboshi_s@niigata-min. or.jp

Cite this article: Hosokawa $\mathrm{H}$, et al. (2022). Association between extended-spectrum $\beta$-lactamase-producing Escherichia coli and oral third-generation cephalosporins. Infection Control \& Hospital Epidemiology, 43: 261-263, https://doi.org/10.1017/ ice.2020.1400
}

To the Editor-The prevalence of extended-spectrum $\beta$-lactamase (ESBL)-producing Escherichia coli is increasing worldwide, and infectious disease caused by ESBL-producing E. coli is associated with increased hospitalization and mortality rates. ${ }^{1,2}$ The high consumption of broad-spectrum oral antibiotics is a serious issue because it likely increases the prevalence of ESBL-producing E. coli. ${ }^{3}$ However, in Japan, these oral antibiotics, which include 
Table 1. Multiple Linear Regression Analysis Between Antibiotic Consumption and the ESBL-Producing E. coli Isolation Ratio

\begin{tabular}{|c|c|c|c|c|c|c|}
\hline Agent & $\mathrm{DDD}^{\mathrm{a}}$ & $\begin{array}{c}P \\
\text { Value }^{b}\end{array}$ & $\mathrm{DOT}^{\mathrm{a}}$ & $\begin{array}{c}P \\
\text { Value }^{\mathrm{b}}\end{array}$ & $\mathrm{DDD} / \mathrm{DOT}^{\mathrm{a}}$ & $\begin{array}{c}P \\
\text { Value }^{b}\end{array}$ \\
\hline First-generation cephalosporins & $-0.006(0.005)$ & .26 & $-0.005(0.004)$ & .33 & $\cdots$ & \\
\hline Third-generation cephalosporins & $\cdots$ & & $-0.0006(0.001)$ & .61 & $-0.72(0.243)$ & .02 \\
\hline Tetracyclines & $\ldots$ & & $-0.001(0.002)$ & .47 & $-0.06(0.079)$ & .49 \\
\hline Penicillins & $-0.002(0.002)$ & .32 & $-0.0005(0.003)$ & .87 & $\cdots$ & \\
\hline Total & $0.0001(0.0002)$ & .43 & $\ldots$ & & $\ldots$ & \\
\hline Adjusted $R^{2}$ & 0.29 & & 0.21 & & 0.45 & \\
\hline
\end{tabular}

Note. ESBL, extended-spectrum beta-lactamase; DDD, defined daily dose; DOT, days of therapy.

aValues shown are partial regression coefficients (standard error); blank spaces indicate values not selected in the model.

${ }^{\mathrm{b}}$ Multiple linear regression analysis.

macrolides, quinolones, and third-generation cephalosporins (3GCs), account for $>90 \%$ of all antibiotic consumption. ${ }^{4}$ Here, we compared the isolation ratio of ESBL-producing E. coli and oral antibiotic consumption in rural Japan.

Data on the characteristics of participating hospitals, amount and days of oral antibiotic prescriptions for outpatients, number of outpatients, and isolation of E. coli for outpatients from January to December 2018 were retrospectively requested from hospitals via the Consortium Against Health Care-Associated Infection in Niigata. This organization is a voluntary organization composed mainly of large-scale hospitals in Niigata Prefecture that aims to improve infection control and prevention. Niigata Prefecture has a population of $\sim 2.2$ million. This study was approved by the Ethics Committee of Kaetsu Hospital (no. 2019-006).

Antibiotics were classified as penicillins, first-generation cephalosporins, 3GCs, macrolides, quinolones, tetracyclines, and other. Antituberculosis agents were excluded. Antibiotics were standardized by the defined daily dose (DDD) per 1,000 patient days and days of therapy (DOT) per 1,000 patient days. The DDD was calculated using the 2018 World Health Organization anatomic therapeutic chemical (ATC)/DDD index. The DDD/DOT ratio was calculated as the DDD value divided by the DOT value. Duplicate isolates of E. coli from the same patient were deleted, and ESBL-producing E. coli were determined according to the screening and confirmation tests suggested by the Clinical and Laboratory Standards Institute. The isolation ratio of ESBL-producing E. coli was calculated as the number of isolated ESBL-producing E. coli divided by the number of all isolated E. coli. ${ }^{2}$

Simple linear regression analysis was performed to examine the relationship between antibiotic consumption and the ESBL-producing $E$. coli isolation ratio. The factors that demonstrated a certain degree of association $(P<0.3)$ were then included as explanatory variables in multiple linear regression analysis. Antibiotics classified as "other" were not included in the multivariate analysis. All analyses were performed with $\mathrm{R}$ version 3.4.1 software ( $\mathrm{R}$ Foundation for Statistical Computing, Vienna, Austria). Correlation coefficients $(R)$ and $P$ values were calculated in the simple linear regression analysis. The partial regression coefficient (standard error) and adjusted $R^{2}$ values were calculated in the multiple linear regression analysis. Statistical significance was set at $P<.05$.

Data were collected from 13 hospitals (41\%) of all 32 hospitals. Table 1 shows only the DDD/DOT ratio of 3GC had a significant negative association with the ESBL-producing $E$. coli isolation ratio (adjusted $\mathrm{R}^{2}=0.45$ ). Supplemental Material 1 shows a summary of the data collected from the 13 participating hospitals. Supplemental Material 2 shows antibiotic consumption and the results of simple linear regression analysis between antibiotic consumption and the ESBL-producing E. coli isolation ratio.

To the best of our knowledge, this is the first report to clarify that the inappropriate dosing of oral 3GCs might be increasing the prevalence of ESBL-producing E. coli. Our results show that only the DDD/DOT ratio of 3GCs had a significant negative association with the isolation ratio of ESBL-producing $E$. coli, indicating that a low daily dose of a $3 \mathrm{GC}$ was associated with an increased ESBL-producing E. coli isolation ratio. In Japan, the approved maintenance dose of antibiotics are often lower than the DDD defined by the World Health Organization. ${ }^{5}$ In addition, the bioavailability of 3GCs is generally low, and the bioavailability of cefcapene and cefditoren, which were mainly used in this study, was reported as $20 \%-30 \%{ }^{6,7}$ Thus, the serum concentration of $3 \mathrm{GC}$ can easily become very low when used at a low dose, and the exposure of bacteria to the mutant selection window of the $3 \mathrm{GC}$ is likely increased. Even though the mutant selection windows of these oral $3 \mathrm{GCs}$ have not been reported, when the dose of cefcapene or cefditoren is reduced, the serum concentration likely reaches the mutant selection window a few hours after administration.

Our study has some limitations, including a small sample size and being a retrospective study conducted in a single region. Additionally, antibiotics for children and adults were assessed without distinction, and elderly patients with suspected renal dysfunction might be administered a reduced dose of antibiotics, which may have influenced the low DDD and DDD/DOT values. Therefore, a larger study is warranted to confirm our findings. Nevertheless, our findings suggest that the inappropriate use of oral $3 \mathrm{GC}$ might be increasing the prevalence of ESBL-producing E. coli.

Supplementary material. To view supplementary material for this article, please visit https://doi.org/10.1017/ice.2020.1400

Acknowledgments. We thank the pharmacists who assisted in data collection to this study.

Financial support. No financial support was provided relevant to this article.

Conflicts of interest. All authors report no conflicts of interest relevant to this article.

\section{References}

1. Karanika S, Karantanos T, Arvanitis M, Grigoras C, Mylonakis E. Fecal colonization with extended-spectrum beta-lactamase-producing Enterobacteriaceae and risk factors among healthy individuals: a systematic review and metaanalysis. Clin Infect Dis 2016;63:310-318. 
2. Chong Y, Shimoda S, Yakushiji H, et al. Community spread of extendedspectrum $\beta$-lactamase-producing Escherichia coli, Klebsiella pneumoniae and Proteus mirabilis: a long-term study in Japan. J Med Microbiol 2013;62:1038-1043.

3. Kimura Y, Fukuda H, Hayakawa K, et al. Longitudinal trends of and factors associated with inappropriate antibiotic prescribing for non-bacterial acute respiratory tract infection in Japan: a retrospective claims database study, 2012-2017. PLoS One 2019;14:1-15.

4. Tsuzuki S, Kimura Y, Ishikane M, Kusama Y, Ohmagari N. Cost of inappropriate antimicrobial use for upper respiratory infection in Japan. BMC Health Serv Res 2020;20(1):153.
5. Muraki Y, Kitamura M, Maeda Y, et al. Nationwide surveillance of anti consumption and resistance to Pseudomonas aeruginosa isolates at 203 Japanese hospitals in 2010. Infection 2013;41:415-423.

6. Tanimura H, Uchiyama K, Onishi $\mathrm{H}$, et al. Study of the absorption of cefcapene pivoxil in patients with infectious disease and soft stool or diarrhea. J Infect Chemother 2003;9:75-82.

7. Sádaba B, Azanza JR, Quetglas EG, et al. Pharmacokinetic/pharmacodynamic serum and urine profile of cefditoren following single-dose and multiple twice- and thrice-daily regimens in healthy volunteers: a phase I study. Rev Esp Quimioter 2007;20:51-60.

\title{
Rational allocation of coronavirus disease 2019 (COVID-19) vaccines to healthcare personnel and patients: A role for antimicrobial stewardship programs?
}

\author{
Priya Nori MD ${ }^{1}$ (1) Payal K. Patel MD, $\mathrm{MPH}^{2}$ and Michael P. Stevens MD, MPH ${ }^{3}$ \\ ${ }^{1}$ Division of Infectious Diseases, Department of Medicine, Montefiore Medical Center, Albert Einstein College of Medicine, Bronx, New York, ${ }^{2}$ Infectious Diseases \\ Section, Ann Arbor Veterans' Affairs Medical Center, Ann Arbor, Michigan and ${ }^{3}$ Healthcare Infection Prevention Department, Virginia Commonwealth University \\ Health System, Richmond, Virginia
}

To the Editor-Vaccine allocation planning is not traditionally considered a core activity of antimicrobial stewardship programs (ASPs). ${ }^{1,2}$ However, ASP physicians and pharmacists are well suited to participate in health-system-wide coronavirus disease 2019 (COVID-19) vaccination efforts given their expertise, programmatic infrastructure, institutional leadership, and trust within their organizations. Although ASPs have played a central role in the COVID-19 response pertaining to novel therapeutics, 3,4 little has been published on ASP involvement in COVID-19 vaccine allocation planning. A PubMed search for "antimicrobial stewardship and severe acute respiratory coronavirus virus 2 (SARS-CoV-2) vaccine" and "antimicrobial stewardship and COVID-19 vaccine" on December 2, 2020, returned no relevant results. Given the rapid expansion of their roles during the pandemic, ASPs may be asked to contribute to health system COVID-19 vaccine allocation planning. ASP contributions to similar institutional efforts predate the pandemic. For instance, ASPs hold important roles in institutional pharmacy and therapeutics committees for formulary addition of new vaccines (eg, new recombinant zoster and meningococcal vaccines). Historically, some ASPs were integrated into 2009 H1N1 influenza pandemic response efforts, participating in vaccine planning and the development of antiviral treatment guidelines. ${ }^{5}$

Since the COVID-19 pandemic began, ASPs have developed significant experience in designing rational allocation systems for novel therapeutics such as remdesivir, ${ }^{4}$ which is now incorporated into ASP preauthorization paradigms throughout the country. ASP pharmacists have particular expertise in preparation,

\footnotetext{
Author for correspondence: Priya Nori, E-mail: pnori@montefiore.org

Cite this article: Nori P, Patel PK, and Stevens MP. (2022). Rational allocation of coronavirus disease 2019 (COVID-19) vaccines to healthcare personnel and patients: A role for antimicrobial stewardship programs?. Infection Control \& Hospital Epidemiology, 43: 263-265, https://doi.org/10.1017/ice.2020.1393
}

transport, and storage required for specific COVID-19 vaccine products. Extreme temperature requirements pose a major challenge for community hospitals and nursing facilities in rural areas without access to medical-grade deep freezers. ${ }^{6}$ ASPs can develop coordinated vaccine distribution systems from a centralized location within health systems to distant facilities. ASPs frequently interface with health information technology departments to design electronic medical record add-ons for stewardship functions, which can be harnessed to create a streamlined order set for all available COVID-19 vaccines and templates for mandatory documentation and reporting required by state health departments.

ASPs have also become a trusted resource for accurate interpretation and dissemination of published data and society guidelines on SARS-CoV-2 therapeutics, such as remdesivir, convalescent plasma, and monoclonal antibodies. Applying this concept to vaccines, ASPs can work with institutional leaders to review data from COVID-19 vaccine trials and adapt recommendations of the Advisory Committee on Immunization Practices (ACIP) on ethical vaccine allocation to their health systems, with the goals of maximizing benefits and minimizing harms, promoting justice and transparency, and mitigating health inequities. ${ }^{7}$ ASPs can also help health systems quickly adapt to evolving guidance from public health authorities in terms of initial target vaccination groups. ${ }^{7}$ Healthcare workers at greatest risk of exposure as well as those caring for the most vulnerable patients will be prioritized in phase 1 of vaccination. ${ }^{7}$ Partnership with local skilled nursing facilities (SNFs) may be required to bolster their resident and associate vaccination efforts. ASP pharmacists and physicians are well suited to provide expertise pertaining to vaccine education, preparation, storage, and monitoring and reporting side effects.

Although ASPs are assets to vaccine allocation planning, many challenges remain. Due to limited initial vaccine doses, a

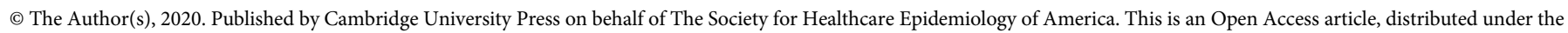
terms of the Creative Commons Attribution licence (http://creativecommons.org/licenses/by/4.0/), which permits unrestricted re-use, distribution, and reproduction in any medium, provided the original work is properly cited. 